\title{
Entre expression collective des citoyens et recomposition des identités individuelles
}

Tourya Guaaybess et Nicolas Pélissier

\section{(2) OpenEdition}

Édition électronique

URL : http://journals.openedition.org/ctd/1687

DOI : $10.4000 /$ ctd. 1687

ISSN : 2491-1437

Éditeur

Chaire Unesco Pratiques émergentes en technologies et communication pour le développement

Édition imprimée

Date de publication : 12 novembre 2015

\section{Référence électronique}

Tourya Guaaybess et Nicolas Pélissier, « Entre expression collective des citoyens et recomposition des identités individuelles ", Communication, technologies et développement [En ligne], 2 | 2015, mis en ligne le 09 juin 2019, consulté le 22 juillet 2020. URL : http://journals.openedition.org/ctd/1687 ; DOI : https://doi.org/10.4000/ctd.1687

Ce document a été généré automatiquement le 22 juillet 2020.

Communication, technologies et développement 


\title{
Entre expression collective des citoyens et recomposition des identités individuelles
}

\author{
Tourya Guaaybess et Nicolas Pélissier
}

\section{Au-delà du déterminisme...}

1

Les mouvements insurrectionnels en Europe Centrale et Orientale qui ont précédé et préparé la chute du mur de Berlin, les soulèvements en Iran en 2009 et plus récemment ceux liés aux «Printemps arabes» ont bien montré que les médias, traditionnels ou numériques, pouvaient représenter des vecteurs privilégiés d'information et d'expression des opposants aux autorités officielles. La littérature récente sur les médias et les mobilisations (Fillieule et alii, 2009; Neveu, 2004; Cardon et Granjon, 2013) donne des clés précieuses pour appréhender les différents types de mouvements sociaux et leurs liens avec ces médias. Ce numéro 2 de la revue Communication, Technologies et Développement se propose de les rapporter à la situation des pratiques contemporaines des TIC sur les cinq continents, de leur apporter une cohérence théorique et de les confronter pour relever les spécificités et les invariants des usages des TIC en période de crise sociale. Aussi, les approches empiriques et comparatives sont-elles ici largement représentées.

2 Selon nombre d'experts, les technologies d'information et de communication ne sont pas tant les acteurs majeurs que des catalyseurs et des révélateurs de dynamiques sociales enracinées dans les cultures concernées. A travers une étude comparée des cas tunisien et sénégalais, Benoît Tine et Mohamed-Ali Elhaou remettent clairement en question tout déterminisme technologique, en même temps qu'ils entendent sortir d'une approche culturaliste des médias.

3 Les historiens des mentalités ont montré la place des rumeurs et de la circulation « virale » de certaines informations stratégiques dans le déclenchement d'événements 
politiques majeurs, mais aucun n'aurait défini les gazouillis de la twittosphère comme les moteurs de ces derniers. Il y a plus de deux décennies, aux temps de la «télérévolution » roumaine ou panarabes, on prêtait déjà volontiers à la télévision et aux chaînes d'information en continu (de même qu'à la radio quelques années plus tôt...) un tel pouvoir messianique (Semelin, 1992; Machcewicz 2015). Soit. Mais la présence et la puissance d'action des TIC, en tout lieu et à chaque instant, ne les positionnent-elles pas désormais au centre des nouvelles stratégies d'action publique?

4 Les récents outils d'information-communication ont été, partout à la fois, associés à des pratiques locales et à des principes universels de liberté, d'émancipation et de partage, mais aussi à un imaginaire d'Internet qui transcende les systèmes de valeurs spécifiques pour les accommoder à des mouvements d'émancipation et à une contreculture faite de bricolages, d'innovations, de témérité, d'insoumission, d'hybridation culturelle. Les auteurs de ce dossier en rendent bien compte. Ainsi, les évènements politiques et médiatiques liés aux grandes mobilisations à travers le monde de cette dernière décennie ont eu cela de troublant qu'ils ont rendu plus visibles que jamais une nouvelle figure héroïque: celle des cyberactivistes expérimentés, non seulement en Europe ou aux Etats-Unis, mais aussi dans des pays où beaucoup ne les attendaient pas (Guaaybess, 2015). En Italie, Paola Sedda démontre que le blog d'un célèbre humoriste, Beppe Grillo, à l'origine du mouvement 5 étoiles, s'avère un véritable espace de contre-pouvoir capable de mobiliser de nombreux internautes attirés par cet espace public oppositionnel. A l'inverse, Yazan Badran et Enrico De Angelis prouvent que certains espaces numériques trop fragmentés ne peuvent faire émerger que des micro-célébrités... comme c'est le cas en Syrie, où le discours des cyberdissidents se heurte au cadre interprétatif dominant du pouvoir.

\section{Rupture numérique et continuités sociopolitiques}

L'objectif de ce travail collectif n'est pas de contribuer au mythe de technologies libératrices des contraintes sociales, géographiques et politiques qui pèsent sur les individus, ni de verser dans la représentation dichotomique de mobilisations vertueuses qui feraient face à des autorités malfaisantes et déconnectées. Sur ce point, il s'inscrit dans une vision critique du paradigme développementaliste qui postule un point de départ négatif constitué par les régimes autoritaires des pays du Sud et de l'Est puis une évolution heureuse et nécessaire, une trajectoire linéaire et inéluctable vers un point d'arrivée construit par le modèle des démocraties libérales du Nord et de l'Ouest (Badie, 2014, Misse Misse 2015). Dans cette transition vers la démocratie, les médias et les TIC joueraient un rôle déterminant.

6 Largement critiquées dans les années 1990, ces théories ont connu un regain d'intérêt avec les récentes mobilisations sociales et politiques relayées par les médias numériques en Afrique, en Asie du Sud-est ou Amérique du Sud (Bonjawo 2002, Olorunnisola et Douai 2013). Elles ont pourtant été sévèrement démenties par les faits, comme l'attestent nombre de travaux récents (Daghmi et alii, 2013, Najar 2013). Dans ce numéro, Sarah Berubé et Olivier Pulvar montrent bien, à l'appui des cas antillais et mexicain, que lors de crises sociales «les médias agissent sur les cultures et les identités au moins autant que ces dernières en réinterprètent les messages pour les faire fonctionner dans un espace social donné ». 
7 Car les TIC sont aussi les instruments contre-révolutionnaires au service d'acteurs économiques hégémoniques ou d'Etats (Morozov, 2011) par ailleurs gestionnaires des infrastructures et contrôlant les opérateurs techniques (fournisseurs d'accès, entreprises nationales de télécommunications, etc.), comme l'illustrent les exemples ukrainien ou syrien ici traités. Les anciens régimes égyptien et tunisien de Moubarak et Ben Ali, pour prendre d'autres cas d'études, obéissaient à des injonctions paradoxales : d'un côté, ces Etats utilisaient les NTIC à des fins de contrôle en réprimant sévèrement les cyberactivistes les plus téméraires ; de l'autre, ils ont favorisé l'accès aux TIC d'une grande partie de la population tunisienne et égyptienne.

8 En tout état de cause, les technologies numériques semblent encourager et renouveler la participation politique. Désormais, des individus et des groupes s'organisent et prennent leur destin en main. Ainsi, explique Abdelouahab Makhloufi, les jeunes Algériens mobilisent les réseaux sociaux pour mener des actions solidaires et civiques au quotidien. Divers types d'actions citoyennes témoignent de cet empowerment. Les usages des TIC traduisent également des revendications diverses, portées par des acteurs aux identités plurielles. Au Cameroun par exemple, l'article de Aboubakar Sidi Njtutammpwoui et Jean-Pierre Ngoulouré montre que les minorités ethniques ou marginalisées peuvent désormais affirmer leurs identités sur des plateformes en ligne toujours plus nombreuses et participer aux débats nationaux, n'attendant qu'un cadre propice à une participation politique plus effective.

9 A vrai dire, les territoires des mobilisations ne sont pas toujours tangibles, leur géographie reste souvent fluide. A l'heure des communautés et des réseaux numériques, les contours de ces territoires réels et symboliques reposent davantage sur des acteurs ancrés ou non dans un espace précis. Les motivations des opposants, des insurgés, des activistes du cyberespace peuvent s'inscrire ou non dans le cadre des Etats-Nations : l'opposition canonique à des régimes autoritaires, si elle est centrale et mérite qu'on s'y arrête, n'est pas la seule cause de mobilisation. D'autres raisons prévalent et qui vont de pair avec l'élargissement de l'espace public rendu possible par les TIC. Elles sont liées à des revendications identitaires ou catégorielles (soulèvement de minorités sexuelles, de groupes ethniques, religieux ou laïcs, des avocats, des jeunes diplômés, etc.) et des protestations pour une meilleure redistribution des ressources. Se dessinent ainsi de nouvelles opinions publiques, nationales ou internationales, incarnées par des manifestants mus par une même cause.

\section{«Ordinaires » de la communication et du journalisme}

Ainsi les TIC suscitent une palette très variée d'expressions politiques et culturelles. Leurs usagers, à la fois producteurs et publics, font preuve de créativité, d'inventivité, d'une capacité à détourner les modalités d'utilisation des outils pour créer du lien social. Jamais la pensée de Michel de Certeau à propos des "ordinaires» de la communication, mais aussi du journalisme (Ruellan 2007), n'aura été autant d'actualité. Photos, dessins, textes ou vidéos circulent de réseau en réseau, élargissent l'espace public, l'enrichissent en autant de lieux d'échange. L'expression citoyenne et de soi y renouvellent de ce fait les identités collectives. Lors de la "Révolution de la Dignité " en Ukraine, les espaces d'expressions politiques et culturelles en ligne ont constitué un prolongement des espaces physiques de la mobilisation. Ces expressions citoyennes 
fonctionnent, comme le démontre Valentyna Dymytrova, dans une dialectique constante avec le journalisme.

11 Ce journalisme numérique, professionnel ou amateur, semble correspondre à un changement de paradigme, spécialement dans des pays où les organes d'Etat constituaient les principales sources d'information. Le journalisme citoyen en ligne au Mozambique en est une parfaite illustration. Maud Pélissier et César Cumbe prouvent ainsi que ce média a son importance aux moments forts de la politique mozambicaine tels que les rendez-vous électoraux. De là à penser que les campagnes électorales sur les médias sociaux sont exemptes de tout vice, il n'y a qu'un pas que Horea Mihai Badau ne franchit pas: dans le cas des dernières élections présidentielles en Roumanie, les contenus des campagnes sur Facebook et Twitter n'ont rien à envier aux exagérations et projections mythologisées des tabloïds les plus populaires.

De plus en plus, l'activité journalistique épouse des contours fuyants et multidimensionnels. Ces changements relèvent de facteurs d'ordre structurel mais aussi conjoncturel qui bousculent les repères des acteurs concernés. Les représentations professionnelles sont confrontées à des logiques politiques, économiques et technologiques qui les resituent dans une configuration systémique où les enjeux du métier ne peuvent plus être appréhendés à partir d'une seule posture épistémologique. Le caractère instable de la strate numérique et la difficulté d'appréhender la panoplie des pratiques au sein d'organisations médiatiques, plurielles dans leur approche de l'information et singulières dans leurs spécificités, sont autant d'éléments qui concourent à complexifier l'étude des transformations induites par les NTIC.

13 Les travaux les plus récents montrent la singularité de la réappropriation des dispositifs socionumériques d'information et de communication par leurs usagers journalistes. Chaque dispositif a son propre mode de fonctionnement, plus ou moins ouvert ou contraignant, qui engendre des usages fort divers, avec des réappropriations individuelles et collectives qui diffèrent avec les cultures et symboliques organisationnelles. Un tel constat redonne toute sa pertinence aux approches sociotechniques des innovations technologiques, très en vogue dans les années 1980-1990 (Latour 1989) mais en relatif recul depuis quelque temps, tant par leur tendance à la dispersion théorique que par le retour en force de visions plus déterministes et globalisantes mentionnées plus haut.

\section{Repenser les liens entre le social et la technique}

Que le modèle choisi soit celui des usages ou des dispositifs, ce sont deux manières de questionner les relations entre social et technique qui ne semblent pas avoir suffisamment discuté ensemble. Ces deux courants de recherche sont nés pourtant au même moment, à la fin des années 1970, dans un contexte d'interrogation portant sur l'autonomie des individus. Depuis, ils semblent avoir évolué en sens inverse: les recherches sur les usages ont cherché à monter progressivement en théorie et dépasser les approches simplement empiriques afin de gagner en épaisseur critique; d'autre part, des travaux sur le dispositif ont tenté transformer un modèle théorique en concept tel que celui de dispositif sociotechnique d'information et de communication susceptible d'organiser les approches empiriques. 
«Le concept de DISTIC oppose un ensemble d'interactions complexes, de technologies et de sujets qui s'en emparent, et non pas tel ou tel élément (les médias, l'internet, le téléphone, les mouvements sociaux...) dont on pourrait isoler les effets mécaniques » (Rasse, Pélissier et Durampart, 2014, pp. 134-135).

Il semble pertinent d'intégrer à ces réflexions les travaux menés dans les champs des Cultural and Gender Studies, mais aussi celui des Science and Technology Studies et des Post-Colonial Studies (Sibeud 2004). Ces derniers se révèlent en effet très féconds pour mieux penser l'insertion sociale des TIC avec une approche globale, comme le fait ici Joëlle Palmieri. Son observation de l'usage des TIC au Sénégal et en Afrique du Sud aboutit à un constat amer : la Société de l'information accentue les inégalités sociales et culturelles, mais aussi les inégalités de genre. Ses analyses rejoignent celle de Victor Sanchez et Juan Manuel Villalbolos dans le sens où ces auteurs, tout en reconnaissant une appropriation des TIC par les mouvements Zapatistes et Pour la Paix, la Justice et la Dignité au Mexique, n'en rappellent pas moins les inégalités en termes d'accès matériel et cognitifs aux médias dans les zones où les infrastructures de bases sont souvent déficientes.

Rasse P., Pélissier N et Durampart M., Les Dispositifs Sociotechniques d'Information et de Communication, un concept transversal, Les Cahiers de la SFSIC, $n^{\circ} 10,2014$.

Ruellan D., Le journalisme ou le professionnalisme du flou, Presses universitaires de Grenoble, 2007

Sémelin J. «Communication et résistance : Les radios occidentales comme vecteur d'ouverture à l'Est ». In: Réseaux, 1992, volume 10 n53. pp. 9-24.

Sibeud E., « Post-Colonial et Colonial Studies : enjeux et débats. ", Revue d'histoire moderne et contemporaine 5/2004 (no51-4bis), p. 87-95

Vidal G (dir), La sociologie des usages. Continuités et transformations, Paris, Hermès Lavoisier, 2012.

\section{BIBLIOGRAPHIE}

\section{BIBLIOGRAPHIE}

Antheaume A., Le journalisme numérique, Paris, Presses de Sciences Po (P.F.N.S.P.) « Nouveaux Débats », 2013

Badie B., Le Temps des Humiliés. Pathologie des relations internationales, Odile Jacob, 2014.

Badillo P., Pélissier N. (dir), Usages et usagers de l'information numérique, dossier de la Revue Françaises des Sciences de l'Information et de la Communication, nº, 2015.

Cardon D., Granjon F., Médiactivistes, Nouvelle édition, Paris, Presses de Sciences Po (P.F.N.S.P.) « Contester ", 2013

Daghmi F. et Alii (dir), Les médias font-ils les révolutions ?, Paris, l'Harmattan, 2013. 
Denouël J., Granjon F. (dir), Communiquer à l'ère numérique. Regards croisés sur la sociologie des usages, Paris, Presses des Mines, 2011.

Fillieule O., Mathieu L., Péchu C., Dictionnaire des mouvements sociaux, Paris, Presses de Sciences Po (P.F.N.S.P.), 2009.

Guaaybess T., «Cadrage journalistique et visibilité des cyberactivistes des « révolutions arabes », in Guaaybes T (dir.). Cadrages journalistiques des révolutions arabes dans le monde, L'Harmattan, 2015.

Jacques B., Internet : Une chance pour l'Afrique, Kharthala, Paris, 2002.

Jauréguiberry F., Proulx S., Usages et enjeux des technologies de communication, Toulouse, Éditions Érès, 2011.

Latour B., La science en action. Introduction à la sociologie des sciences. Paris : La Découverte, 1989.

Machcewicz, Paweł. Poland's War on Radio Free Europe, 1950-1989, Stanford University Press, 2015

Misse M., «Les théories critiques en communication pour le changement social : regard critique ", Revue française des sciences de l'information et de la communication [En ligne], 4 | 2014, mis en ligne le 01 janvier 2014, consulté le 19 septembre 2015. URL : http://

rfsic.revues.org/931

Morozov E., The Net Delusion: the dark side of Internet freedom, Kindle Editions, New York, 2012. Najar Sihem (dir), Le cyberactivisme au Maghreb et dans le monde arabe, IRMC/Karthala, Paris, 2013.

Neveu E., Sociologie des mouvements sociaux, Paris, La Découverte, 2004.

Olorunnisola A., Douai A., New Media Influence on Social and Political Change in Africa, IGI Global, 2013.

Palmer M., « Transnationales de l'information », Questions de communication, 15 | 2009, 345-366.

Pignard-Cheynel N., Mercier, A., (juin 2014) « Renouvellements des pratiques journalistiques à l'ère du numérique : un état des travaux ", Revue française des sciences de l'information et de la communication.

\section{RÉSUMÉS}

Sans céder au déterminisme technologique qui distingue l'espace social de son avatar numérique et sans souscrire par ailleurs aux discours triomphalistes et euphorisants sur la prétendue révolution 2.0, il devient difficile d'ignorer la place croissante des technologies de l'information et de la communication dans les économies en développement, en accord avec le tempo croissant des breaking news de l'information mondialisée (Michael Palmer, 2009). Mais si la production académique sur la pratique des TIC dans ces pays est de plus en plus abondante, elle se fait plus rare sur la thématique de ce numéro, centré sur les liens entre TIC et mobilisations. Il s'agit ici d'interroger la place et les usages des médias numériques (notamment internet et téléphonie mobile) dans le contexte de mouvements sociaux ou de protestations populaires. 
AUTEURS

TOURYA GUAAYBESS

Université de Metz

NICOLAS PÉLISSIER

Université de Nice Sophia-Antipolis 